\title{
Stent-Assisted Coiling Versus Balloon Remodeling of Wide-Neck Aneurysms: Comparison of Angiographic Outcomes
}

N. Chalouhi, R.M. Starke, M.T. Koltz, P.M. Jabbour, S.I. Tjoumakaris, A.S. Dumont, R.H. Rosenwasser, S. Singhal, and L.F. Gonzalez

\begin{abstract}
BACKGROUND AND PURPOSE: Stent-assisted coiling and balloon-assisted coiling are 2 well-established techniques for treatment of wide-neck intracranial aneurysms. A direct comparative analysis of angiographic outcomes with the 2 techniques has not been available. We compare the angiographic outcomes of wide-neck aneurysms treated with stent-assisted coiling versus balloon-assisted coiling.
\end{abstract}

MATERIALS AND METHODS: A retrospective review was conducted on 101 consecutive patients treated at our institution, 69 with stent-assisted coiling and 32 with balloon-assisted coiling. Two multivariate logistic regression analyses were performed to determine predictors of aneurysm obliteration and predictors of progressive aneurysm thrombosis at follow-up.

RESULTS: The 2 groups were comparable with respect to all baseline characteristics with the exception of a higher proportion of ruptured aneurysms in the balloon-assisted coiling group (65.6\%) than in the stent-assisted coiling group $(11.5 \%, P<.001)$. Procedural complications did not differ between the stent-assisted coiling group (6\%) and the balloon-assisted coiling group $(9 \%, P=.5)$. The rates of complete aneurysm occlusion (Raymond score 1) at the most recent follow-up were significantly higher for the stent-assisted coiling group (75.4\%) compared with the balloon-assisted coiling group ( $50 \%, P=.01)$. Progressive occlusion of incompletely coiled aneurysms was noted in $76.6 \%$ of aneurysms in the stent-assisted coiling group versus $42.8 \%$ in the balloon-assisted coiling group $(P=.02)$. Retreatment rates were significantly lower with stent-assisted coiling $(4.3 \%)$ versus balloon-assisted coiling $(15.6 \%, P=.05)$. In multivariate analysis, stented aneurysms independently predicted both complete aneurysm obliteration and progression of occlusion.

CONCLUSIONS: Stent-assisted coiling may yield lower rates of retreatment and higher rates of aneurysm obliteration and progression of occlusion at follow-up than balloon-assisted coiling with a similar morbidity rate.

ABBREVIATIONS: SAC = stent-assisted coiling; BAC = balloon-assisted coiling; $\mathrm{PED}=$ Pipeline Embolization Device

W ide-neck intracranial aneurysms were classically considered unsuitable for endovascular treatment and were typically managed by surgical clipping. Recent advancements in endovascular devices and techniques have placed such aneurysms within the range of endovascular therapy. Specifically, stent-assisted coiling and balloon-assisted coiling (also known as the balloon remodeling technique) have emerged as safe and efficient treatment modalities for complex and wide-neck aneurysms. Both techniques have improved

Received October 26, 2012; accepted after revision January 20, 2013.

From the Department of Neurological Surgery (N.C., R.M.S., M.T.K., P.M.J., S.I.T., A.S.D., R.H.R., S.S., L.F.G.), Thomas Jefferson University and Jefferson Hospital for Neuroscience, Philadelphia, Pennsylvania; and Department of Neurological Surgery (R.M.S.), University of Virginia, Charlottesville, Virginia.

Please address correspondence to L. Fernando Gonzalez, MD, Department of Neurological Surgery, Division of Neurovascular Surgery and Endovascular Neurosurgery, Thomas Jefferson University Hospital, 901 Walnut St, 3rd Floor, Philadelphia,

PA 19107; e-mail: fernando.gonzalez@jefferson.edu

http://dx.doi.org/10.3174/ajnr.A3538 the long-term durability of endovascular coiling. ${ }^{1,2}$ By preventing coil herniation into the parent vessel and helping the coil to assume the $3 \mathrm{D}$ shape of the aneurysm, the use of a balloon allows denser aneurysm packing than does conventional coiling. ${ }^{3,4}$ On the other hand, a stent acts as a scaffold for coils and improves neck coverage, thus allowing optimal aneurysm coiling. ${ }^{5}$ A growing but currently incomplete body of evidence also suggests that self-expanding stents may have flow-remodeling effects that lead to more durable aneurysm obliteration. ${ }^{2,6,7}$ Although the safety and efficacy of SAC and BAC have been studied in several series, to our knowledge a direct comparative analysis of angiographic outcomes with the 2 techniques has not been available.

We present the results of the first study comparing the angiographic outcomes of BAC and SAC in wide-neck intracranial aneurysms. Two multivariate logistic regression analyses were conducted to determine predictors of aneurysm obliteration and predictors of progressive aneurysm thrombosis at follow-up. 

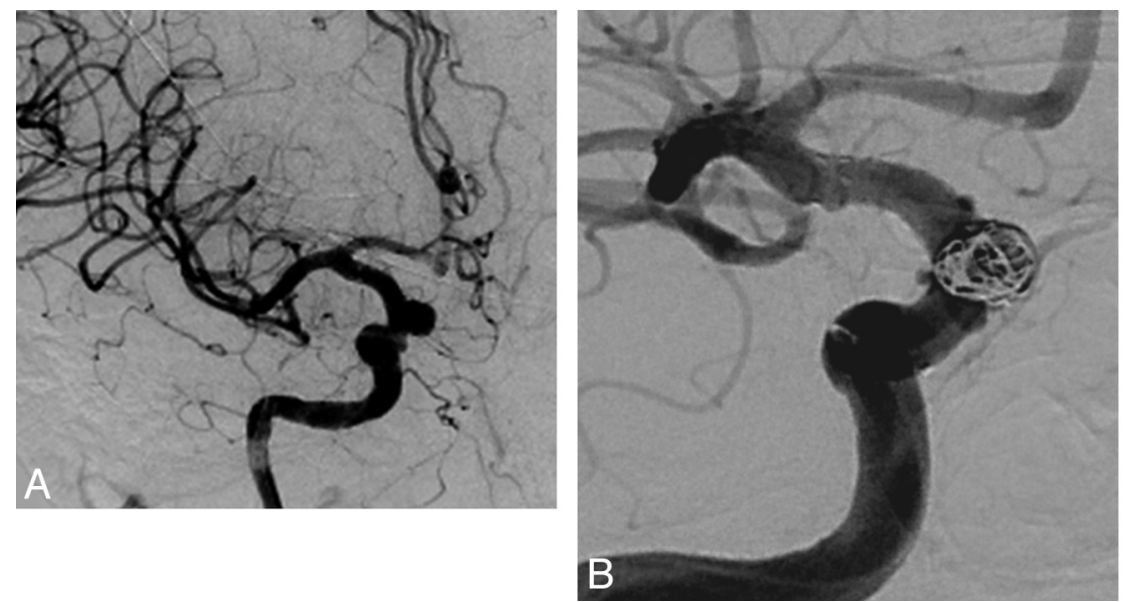

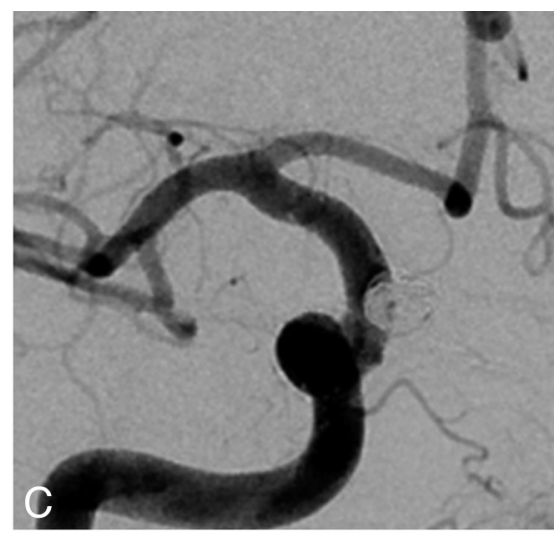

FIG 1. Frontal views of DSA. A, Unruptured wide-neck superior hypophyseal artery aneurysm before treatment. $B$, Angiogram obtained immediately after SAC with residual filling of the aneurysm. C, Twelve-month follow-up angiogram shows progression of the aneurysm to complete occlusion.

\section{MATERIALS AND METHODS}

Institutional review board approval was obtained before data collection. Nine hundred fifty-nine aneurysms were treated (779 with endovascular means, 180 with surgical clipping) at Jefferson Hospital for Neuroscience between 2009-2010. Consecutive patients with wide-neck aneurysms treated by SAC during a 1-year period (2009) or BAC during a 2-year period (2009-2010) at our institution were identified. Wide-neck aneurysms were defined as aneurysms with a fundus-to-neck ratio of $<2$ or a neck diameter of $\geq 4 \mathrm{~mm}$. One hundred twenty-three patients with wide-neck aneurysms were treated with SAC and 57 patients were treated with BAC. Patients with at least 1 available DSA follow-up were included in the study. Previously treated aneurysms were excluded from the analysis. Fifty patients had only MRA/CTA follow-up, 16 patients had previously treated aneurysms, and 13 patients were lost to follow-up. Thus, a total of 101 patients, 69 treated with SAC and 32 treated with BAC, met our criteria and constituted our study population (Fig 1).

Demographic data on patient age, sex, aneurysm location, ruptured or unruptured aneurysm status, aneurysm size, and type of procedure were recorded. Aneurysm volume and packing density were calculated by use of Angiocalc software (http://www. angiocalc.com) on the basis of the angiographic shape and size of the aneurysm as well as the type and size of coils. Initial and follow-up angiograms were reviewed by an independent observer. Aneurysm occlusion was categorized based on the Raymond scale as complete occlusion (Raymond class 1), residual neck (Raymond class 2), and dome filling (Raymond class 3). The incidence of progression of occlusion with BAC versus SAC was determined in aneurysms with an initial Raymond class of 2 or 3.

Procedures were performed under general anesthesia; continuous neurophysiologic monitoring including somatosensory evoked potentials, brain stem auditory-evoked responses, and electroencephalography. Patients with unruptured aneurysms treated with BAC were heparinized, and activated clotting time was maintained intraoperatively at twice the baseline. Patients presenting with $\mathrm{SAH}$ received $35 \mathrm{U} / \mathrm{kg}$ of heparin after deployment of the first coil. Access was obtained with a single $7 \mathrm{~F}$ sheath and a $6 \mathrm{~F}$ guide catheter. A 3-way rotatory hemostatic valve was used in all cases; 3D reconstruction was obtained to best demonstrate the aneurysm neck and the parent vessel. The balloon was advanced through the center port of the rotatory hemostatic valve and left spanning the neck of the aneurysm. Subsequently, a microcatheter was introduced to the aneurysm and an attempt was made initially to place the coils with the balloon deflated. If the coils compromised the lumen of the parent vessel, the balloon was inflated to provide assistance. Multiple coils were deployed during a single inflation. Once coiling had been completed, the balloon was deflated on a blank roadmap to determine the stability of the coil mass. If coil displacement occurred, the balloon was reinflated and more coils were deployed. Antiplatelet therapy was not given in any of these patients either during or after surgery. A HyperGlide (ev3, Irvine, California) balloon was used in 30 patients and a HyperForm (ev3) balloon in 2 patients.

SAC procedures were either staged $(n=35)$ or performed during a single session $(n=34)$ at the operator's discretion. In staged procedures, the stent was placed first across the aneurysm neck and left to endothelialize for 6-12 weeks before the patient was brought back for coil embolization. This usually allowed greater stability and less movement of the stent during coil deployment. Single-stage SAC was performed by use of the microcatheter "jailing" technique. Neuroform stents were sized on the basis of the largest diameter of the target vessel and were usually slightly oversized. When the use of a stent was anticipated, patients were pretreated with $81 \mathrm{mg}$ of aspirin and $75 \mathrm{mg}$ of clopidogrel 10 days before the procedure. Platelet function tests were performed with aspirin assay and P2Y12 assay (VerifyNow; Accumetrics, San Diego, California). Patients treated in the setting of SAH were loaded intraoperatively with $600 \mathrm{mg}$ of clopidogrel via a nasogastric tube immediately after stent deployment. During the procedure, they also received boluses of intravenous heparin to maintain an activated clotting time of 2 to 3 times their baseline. All patients were maintained on daily doses of $75 \mathrm{mg}$ of clopidogrel and $81 \mathrm{mg}$ of aspirin for 2 months, followed by aspirin $81 \mathrm{mg}$ daily indefinitely. The Neuroform stent (Stryker Neurovascular, Fremont, California) was used in 49 patients and the Enterprise stent (Codman \& Shurtleff, Raynham, Massachusetts) in 20 
Table 1: Baseline characteristics of the 2 groups

\begin{tabular}{lccc}
\hline & $\begin{array}{c}\text { Stent } \\
\text { Group }\end{array}$ & $\begin{array}{c}\text { Balloon } \\
\text { Group }\end{array}$ & $\begin{array}{c}\boldsymbol{P} \\
\text { Values }\end{array}$ \\
\hline Age, $y$ & 54.2 & 53.7 & .4 \\
Female sex, $n(\%)$ & $57 / 69(82.6)$ & $26 / 32(81.2)$ & .9 \\
Ruptured aneurysms, $n(\%)$ & $8 / 69(11.5)$ & $21 / 32(65.6)$ & $<.001$ \\
Anterior circulation aneurysms, & $58 / 69(84.1)$ & $26 / 32(81.3)$ & .8 \\
$\quad n(\%)$ & & & \\
Mean aneurysm size, $\mathrm{mm}$ & 6.8 & 6.4 & .3 \\
\hline
\end{tabular}

patients. The choice between Neuroform and Enterprise stent placement was mainly based on operator preference.

Bare platinum coils were used for aneurysm embolization. In BAC, coiling was interrupted when the aneurysm was completely occluded or when the microcatheter was pulled out of the aneurysm. Because of the potential flow-remodeling effects of selfexpanding stents, we may tend to achieve lower packing densities in SAC than in BAC at the operator's discretion. Angiographic follow-up is scheduled at 6 months, 1 year, 2 years, and 5 years after treatment with BAC or SAC at our institution.

\section{Statistical Analysis}

Data are presented as mean and range for continuous variables and as frequency for categoric variables. Analysis was carried out with the use of unpaired $t$ test, $\chi^{2}$, and Fisher exact tests as appropriate.

Univariable analysis was used to test covariates predictive of aneurysm obliteration at follow-up: Raymond score 1 versus 2-3 and Raymond score 1-2 versus 3. Interaction and confounding was assessed through stratification and relevant expansion covariates. Factors predictive in univariable analysis $(P<.15)^{8}$ were entered into a multivariable logistic regression analysis. $P$ values of $\leq .05$ were considered statistically significant. Statistical analysis was carried out with STATA 10.0 (StataCorp, College Station, Texas).

A similar statistical analysis was conducted to determine predictors of progression of occlusion. Only aneurysms that were initially incompletely occluded (Raymond score of 2 or 3 ) were included in this analysis: 47 in the SAC group and 21 in the BAC group (Fig 1).

\section{RESULTS}

\section{Anatomic Results}

The 2 groups were comparable with respect to all baseline characteristics (Table 1) with the exception of a higher proportion of ruptured aneurysms in the BAC group (65.6\%) than in the SAC group $(11.5 \%, P<.001)$.

In the SAC group, Raymond scores were 1 in $22(31.9 \%)$ patients, 2 in $44(63.8 \%)$ patients, and 3 in $3(4.3 \%)$ patients. In the BAC group, Raymond scores were 1 in 11 (34.4\%) patients and 2 in $21(65.6 \%)$ patients $(P=.8)$. Packing denstiy was significantly higher in the BAC (29.4\%) versus the SAC group $(17.5 \%, P<$ .001). Of note, in the SAC group, mean packing density was $23.4 \%$ in ruptured aneurysms and $16.7 \%$ in unruptured aneurysms $(P=$ .3). Mean angiographic follow-up was $10.2 \pm 7.0$ months in the entire cohort (median, 8; range, 3-48), $10.7 \pm 7.5$ months in the SAC group (median, 8 ; range, $5-48$ ), and $8.9 \pm 5.7$ months (median, 6.5; range, $3-36)$ in the BAC group $(P=.2)$. At follow-up, in the SAC group, Raymond scores were 1 in 52 (75.4\%) patients, 2 in $13(18.8 \%)$ patients, and 3 in $4(5.8 \%)$ patients. In the BAC group, Raymond scores were 1 in $16(50 \%)$ patients, 2 in 11 $(34.4 \%)$ patients, and 3 in $5(15.6 \%)$ patients. The rates of complete aneurysm occlusion (Raymond 1) at follow-up were significantly higher for the SAC group (75.4\%) compared with the BAC group $(50 \%, P=.01)$. The rates of adequate aneurysm occlusion (Raymond 1-2) at follow-up, however, did not differ significantly between the SAC group (94.2\%) and the BAC group (84.4\%, $P=$ .1). In univariate analysis, the following factors were tested for as predictors of complete obliteration (Raymond 1$)$ : age $(P=.7)$, sex $(P=.5)$, ruptured/unruptured aneurysm status $(P=.8)$, aneurysm location $(P=.005)$, aneurysm size $(P=.03)$, packing density $(P=.2)$, follow-up duration $(P=.5)$, initial aneurysm occlusion $(P=.6)$, and type of treatment $(P=.01)$. Aneurysm location, aneurysm size, and type of treatment were subsequently entered into a multivariate model. In multivariate analysis, smaller $(<7 \mathrm{~mm})$ aneurysms $(\mathrm{OR}=0.83$; 95\% CI, 0.68-0.98; $P=.01)$ and stented aneurysms $(\mathrm{OR}=0.3 ; 95 \% \mathrm{CI}, 0.12-0.81$; $P=.02$ ) were independent predictors of complete obliteration at follow-up. Posterior communicating artery-carotid cavernouscarotid terminus-middle cerebral artery-posterior carotid wall aneurysms (ie, aneurysm locations with rates of complete occlusion below 60\%) $(\mathrm{OR}=3.3$; 95\% CI, 1.3-8.6; $P=.01)$ were negative predictors of obliteration. These 3 factors remained statistically significant even after controlling for packing density and ruptured aneurysm status. The same factors as above were tested for as predictors of adequate aneurysm occlusion (Raymond $1-2)$. Aneurysm size $(P=.01)$ and stented aneurysms $(P=.1)$ were the only predictors in univariate analysis. In multivariate analysis, smaller $(<7 \mathrm{~mm})$ aneurysms ( $\mathrm{OR}=0.2 ; 95 \% \mathrm{CI}, 0.06-$ $0.6 ; P=.004)$ were predictive of adequate aneurysm occlusion. There was also a trend for stented aneurysms ( OR $=0.2 ; 95 \% \mathrm{CI}$, $0.05-1.2 ; P=.08)$ to predict adequate aneurysm occlusion.

The rates of retreatment were $4.3 \%(3 / 69)$ in the SAC group versus $15.6 \%(5 / 32)$ in the BAC group $(P=.05)$. No patient in the SAC group had evidence of in-stent stenosis or occlusion on follow-up angiograms.

Of 47 aneurysms in the SAC group and 21 aneurysms in the BAC group that were initially incompletely occluded, progressive occlusion at follow-up was noted in $36(76.6 \%)$ aneurysms in the SAC group and $9(42.8 \%)$ aneurysms in the BAC group $(P=.02)$. In univariate analysis, the following factors were tested for as predictors of progression of occlusion: age $(P=.8)$, sex $(P=.2)$, ruptured/unruptured aneurysm status $(P=.4)$, aneurysm location $(P=.06)$, aneurysm size $(P=.02)$, packing density $(P=.04)$, follow-up duration $(P=.7)$, and type of treatment $(P=.02)$. Aneurysm location, aneurysm size, packing density, and type of treatment were subsequently entered into a multivariate model. In multivariate analysis, decreasing packing density $(\mathrm{OR}=1.03$; $95 \% \mathrm{CI}, 0.9-1.12 ; P=.382)$ and decreasing aneurysm size (OR $=$ $0.9 ; 95 \% \mathrm{CI}, 0.7-1.1 ; P=.2$ ) were no longer predictive of progression of occlusion. Stented aneurysms (OR $=0.08$; 95\% CI, $0.02-$ $0.3 ; P<.001)$ positively predicted progression of occlusion, whereas anterior communicating artery-carotid cavernous-carotid terminus aneurysms (ie, aneurysm locations with incidence of progressive occlusion below 50\%) were negative predictors 
$(\mathrm{OR}=11.5 ; 95 \% \mathrm{CI}, 2.2-59 ; P=.004)$. These 2 factors remained statistically significant even after controlling for packing density and ruptured aneurysms.

\section{Procedural Outcomes}

Procedural complications occurred in $4(6 \%)$ patients of the SAC group and resulted in permanent morbidity in 2 (3\%): Intraoperative aneurysm rupture causing intraparenchymal hemorrhage; new infarct in the posterior limb of right internal capsule; new frontal and parietal infarcts; and small clinically silent parietal infarcts. In the BAC group, there were $3(9 \%, P=.5)$ procedural complications, leaving permanent morbidity in $1(3 \%)$ : intraoperative aneurysm rupture rapidly controlled by balloon inflation and coil packing; thrombus visualized intraprocedurally treated by intra-arterial infusion of abciximab; and hemiparesis caused by a frontal paramedian infarct.

Favorable outcomes (mRS 0-2) at the most recent follow-up visit (mean, 7.3 months) were noted in 66 of 69 patients (95\%) of the SAC group and 29 of 32 patients (91\%) of the BAC group $(P=.9)$.

\section{DISCUSSION}

The most important findings of the present study are the following: 1) SAC of wide-neck aneurysms provides higher rates of complete obliteration (Raymond 1) at follow-up than does BAC. The rates of adequate aneurysm occlusion (Raymond 1-2), however, did not significantly differ between the 2 techniques. 2) Lower retreatment rates were observed with SAC versus BAC in wideneck aneurysms. 3) SAC causes delayed occlusion of incompletely coiled wide-neck aneurysms at a significantly higher rate than does BAC. 4) Both treatment strategies are associated with low rates of morbidity and excellent clinical outcomes.

The high rate of aneurysm recanalization is considered the major drawback of endovascular therapy. Raymond et $\mathrm{al}^{9}$ found some degree of recurrence in as many as $33.6 \%$ of coiled aneurysms and identified aneurysm size $\geq 10 \mathrm{~mm}$, treatment during the acute phase of subarachnoid hemorrhage, incomplete initial occlusion, and duration of follow-up as significant predictors of recurrence. In the International Subarachnoid Aneurysm Trial retreatment was done 4.6 times more commonly after endovascular therapy $(17.4 \%)$ than after microsurgical clipping $(3.8 \%) .{ }^{10,11}$ The risk of late rebleeding was also higher after endovascular repair $(2.9 \%)$ than after open surgery $(0.9 \%)$. In a systematic review of the literature that included 8161 coiled aneurysms, Ferns et $\mathrm{al}^{12}$ reported aneurysm reopening in $20.8 \%$ of cases and retreatment in $10.3 \%$ and identified location in the posterior circulation and size $>10 \mathrm{~mm}$ as risk factors for aneurysm reopening. The adjunctive use of endovascular devices such as nondetachable balloons or self-expanding stents has notably improved the long-term durability of endovascular treatment. As such, in a large study, Piotin et $\mathrm{al}^{2}$ found remarkably lower angiographic recurrence rates with stent-assisted coiling compared with conventional coiling (14.9\% versus $33.5 \%$, respectively). In a recent large multicenter French study (CLARITY), Pierot et $\mathrm{al}^{1}$ identified 3 factors associated with the quality of midterm aneurysm occlusion after endovascular therapy, namely, neck size, the use of the balloon remodeling technique, and the quality of postoperative occlusion. The authors concluded that future treatment strategies should be focused on reinforcement and neoendothelialization at the level of the neck, especially for wide-necked aneurysms.

Initial patient comparability is a significant limitation of studies comparing SAC or BAC with conventional coiling, because aneurysms treated with SAC or BAC tend to be wide-neck, larger, and typically more complex than those treated with conventional coiling. In contrast to previous studies, the present study has the advantage of comparing 2 homogeneous groups of patients harboring wide-neck aneurysms treated by BAC or SAC. Our results are in line with the findings of recent reports showing that stented aneurysms have better angiographic outcomes than nonstented aneurysms coiled with or without balloon assistance. Lawson et $\mathrm{al}^{13}$ compared the follow-up angiographic outcomes of 37 stented versus 72 nonstented (with or without balloon assistance) incompletely coiled aneurysms and found that the odds of progression of occlusion of stent-coiled aneurysms were 18.5 times that of nonstented aneurysms. The authors attributed their results, at least in part, to the "flow remodeling" effects of self-expanding stents. In fact, stents may elicit a neointimal response that leads to endovascular vessel reconstruction and ultimately excludes the aneurysm from the circulation. Lopes and Sani ${ }^{14}$ described complete endothelialization of a Neuroform stent 4 months after its placement in an explanted human aneurysm specimen, with significant de novo fibroelastic tissue formation along the neck of the aneurysm. Such in-growth of endothelial tissue over the stented segment may potentially alter intra-aneurysmal flow dynamics causing stasis and thrombosis inside the aneurysm. This could explain why SAC yields higher rates of aneurysm occlusion and progressive thrombosis than conventional coiling or even BAC. Because of this potential flow-remodeling effect of self-expanding stents, we generally avoid overpacking stented aneurysms and may settle for more modest packing densities. At most neurovascular centers, however, the same range of packing density is achieved in aneurysms coiled with or without stent assistance. In vitro studies have also shown that higher packing densities are associated with significant flow reduction at the neck of the aneurysm. ${ }^{15}$ In addition, obtaining high packing densities remains a priority in ruptured aneurysms treated with SAC to minimize the risk of rebleeding. As such, packing density was $23.4 \%$ on average in ruptured aneurysms versus $16.7 \%$ in unruptured aneurysms.

The main limitation of BAC remains the inability to pack the neck of the aneurysm, because $65 \%$ of aneurysms initially and $35 \%$ at follow-up showed a neck remnant in the present series. This translated into significantly lower rates of complete aneurysm occlusion (Raymond 1) and higher rates of retreatment with BAC versus SAC. The difference between the 2 groups, however, was less prominent when comparing the rates of adequate aneurysm occlusion (Raymond 1-2 versus 3 ), with only a trend favoring SAC in multivariate analysis. Both techniques appear to have high efficacy with respect to adequate aneurysm occlusion at follow-up (94.2\% with SAC and $84.4 \%$ with BAC).

Assessment of procedural safety and clinical outcomes are essential to determine the best therapeutic approach for wide-neck aneurysms. In our study, both techniques were associated with low morbidity rates (3\%) and excellent clinical outcomes $(>90 \%)$. It is worth noting that the significantly higher propor- 
tion of patients treated in the setting of subarachnoid hemorrhage in the BAC group has probably favored the stent group. In fact, the rate of procedural complications with BAC is reportedly $11.7 \%$ in unruptured aneurysms versus $17 \%$ in ruptured aneurysms. ${ }^{4,16}$ Likewise, stent procedures in ruptured aneurysms carry a higher rate of morbidity than in unruptured aneurysms. ${ }^{17}$ For many neurointerventionalists, BAC is preferred over SAC for treatment of ruptured wide-necked aneurysms because the patient is spared the initiation of an antiplatelet regimen and its potential hemorrhagic complications. ${ }^{17}$ Mocco et al ${ }^{17}$ reported a $12 \%$ procedural mortality rate with SAC of acutely ruptured aneurysms and concluded that extreme caution should be exercised when considering stent deployment in patients with subarachnoid hemorrhage. Amenta et $\mathrm{al}^{18}$ treated 65 patients with acutely ruptured aneurysms with a $15.4 \%$ major complication rate including bleeding secondary to antiplatelet therapy in $7.7 \%$ and intraoperative in-stent thrombosis in $7.7 \%$. In a systematic review of the literature of acutely ruptured intracranial aneurysms (339 patients) treated with stents, Bodily et $\mathrm{al}^{19}$ found that intracranial hemorrhagic and thromboembolic complications occurred in $8 \%$ and $6 \%$ of patients, respectively, and concluded that adverse outcomes were more common with SAC compared with conventional coiling. The use of a stent may also have considerable influence on flow in perforating vessels near treated cerebral aneurysms. ${ }^{15}$ On the other hand, a balloon may protect a sidebranch from coil herniation and, in the event of intraprocedural aneurysm rupture, provide proximal control thus preventing devastating consequences for the patient. In a critical review of the literature, Pierot et $\mathrm{al}^{20}$ noted that all except 1 publication showed a similar safety profile between BAC and conventional coiling, with better initial and follow-up anatomic results in aneurysms treated by BAC. On the basis of these data, they recommended wide use of BAC for treatment of wide-neck aneurysms and cautioned against wide use of stents given the potential associated morbidity.

Some operators advocate staging the stent-coil procedure to allow endothelialization of the stent. This may allow for greater stability and less movement of the stent and may also avoid endothelial damage and platelet activation during coil deployment. Accordingly, Lodi et $\mathrm{al}^{21}$ compared single-stage $(n=37)$ and multistage $(n=50)$ SAC and found higher complication rates with single-stage SAC. On the other hand, staging the stent-coil procedure may carry a theoretic risk of interval aneurysm rupture and loss to follow-up with an increased procedural cost. Currently, both techniques are widely applied in clinical practice.

Flow diversion and flow disruption are potential alternatives in the treatment of wide-neck intracranial aneurysms. In a large series of 191 patients with 251 aneurysms treated with the Pipeline Embolization Device (PED) (Covidien, Irvine, California), Saatci et $\mathrm{al}^{22}$ reported an event rate of $14.1 \%$, with a permanent morbidity rate of $1 \%$ and mortality rate of $0.5 \%$. The rate of aneurysm occlusion was $91.2 \%$ at 6 months and further increased to $94.6 \%$ with longer follow-up. In a prospective study from Hong Kong that included 143 patients with 178 aneurysms treated with the $\mathrm{PED}$, Yu et $\mathrm{al}^{23}$ noted periprocedural death or major stroke in $3.5 \%$ of cases with complete aneurysm occlusion in $84 \%$ at 18 months. The authors concluded that PED therapy was safe and effective and should be considered a first choice for treating un- ruptured aneurysms and recurrent aneurysms after previous treatments. Flow disruption with the WEB device (Sequent Medical, Palo Alto, California) is a new technique that has shown promising results for treatment of wide-neck aneurysms. Lubicz et $\mathrm{al}^{24}$ have recently treated 19 patients with 20 unruptured wideneck bifurcation aneurysms by WEB placement and reported symptomatic complications in 2 patients. Although additional stent placement and/or coiling was necessary in 3 cases at the acute phase and in 1 case at follow-up, complete or near-complete aneurysm occlusion was achieved in $89 \%$ of cases at 6 months. The results of this series showing feasibility of treatment of wideneck aneurysms with the WEB require confirmation in larger studies.

The limitations of the present study are related to its retrospective design, the relatively short angiographic follow-up time (10.2 months), and the relatively small sample size, especially in the BAC group. Follow-up time was slightly longer in the SAC group, which may have influenced the anatomic results, especially the rate of progression of occlusion. Ruptured aneurysms were also more common in the BAC group, which may have favored better results in the SAC group, because it is well known that recurrences are more common when treatment is undertaken in the acute setting of subarachnoid hemorrhage. As such, ruptured aneurysms were strongly predictive of aneurysm recurrence in the studies by Piotin et $\mathrm{al}^{2}$ and Raymond et al. ${ }^{9}$ Likewise, Cognard et $\mathrm{al}^{25}$ found that unruptured aneurysms of $<9 \mathrm{~mm}$ have a recanalization rate of $7 \%$ as opposed to ruptured aneurysms of the same size, which had a recurrence rate of $17 \%$. Also, stent-assisted coiling of ruptured aneurysms has been shown to be associated with lower angiographic obliteration rates at follow-up compared with unruptured aneurysms. ${ }^{7}$ Nevertheless, a multivariate analysis was conducted to account for such differences in baseline characteristics and identify independent predictors of occlusion. Prospective, randomized trials will be needed to provide definitive information on the best therapeutic approach for wide-neck aneurysms.

\section{CONCLUSIONS}

In this study, we found that SAC yields lower rates of retreatment and higher rates of complete aneurysm obliteration and progression of occlusion at follow-up than does BAC. The rates of adequate aneurysm occlusion, however, did not differ significantly between the 2 groups. Both techniques appear to have a favorable safety-efficacy profile and may be applied for treatment of wideneck aneurysms. The findings of this retrospective study require confirmation by larger prospective studies.

Disclosures: Pascal Jabbour-UNRELATED: Consultancy: ev3, CNV. Stavropoula Tjoumakaris—UNRELATED: Consultancy: Stryker. Aaron Dumont-UNRELATED: Consultancy: ev3.

\section{REFERENCES}

1. Pierot L, Cognard C, Anxionnat R, et al. Endovascular treatment of ruptured intracranial aneurysms: factors affecting midterm quality anatomic results: analysis in a prospective, multicenter series of patients (CLARITY). AJNR Am J Neuroradiol 2012;33:1475-80

2. Piotin M, Blanc R, Spelle L, et al. Stent-assisted coiling of intracranial aneurysms: clinical and angiographic results in 216 consecutive aneurysms. Stroke 2010;41:110-15

3. Pierot L, Cognard C, Anxionnat R, et al. Ruptured intracranial 
aneurysms: factors affecting the rate and outcome of endovascular treatment complications in a series of 782 patients (CLARITY study). Radiology 2010;256:916-23

4. Pierot L, Cognard C, Anxionnat R, et al. Remodeling technique for endovascular treatment of ruptured intracranial aneurysms had a higher rate of adequate postoperative occlusion than did conventional coil embolization with comparable safety. Radiology 2011; 258:546-53

5. Bendok BR, Parkinson RJ, Hage ZA, et al. The effect of vascular reconstruction device-assisted coiling on packing density, effective neck coverage, and angiographic outcome: an in vitro study. $\mathrm{Neu-}$ rosurgery 2007;61:835-40

6. Izar B, Rai A, Raghuram K, et al. Comparison of devices used for stent-assisted coiling of intracranial aneurysms. PLoS One 2011; 6:e24875

7. Chalouhi N, Dumont AS, Hasan D, et al. Is packing density important in stent-assisted coiling? Neurosurgery 2012;71:381-87

8. Altman D. Practical Statistics for Medical Research. Boca Raton, Florida: Chapman \& Hall/CRC; 1999

9. Raymond J, Guilbert F, Weill A, et al. Long-term angiographic recurrences after selective endovascular treatment of aneurysms with detachable coils. Stroke 2003;34:1398-403

10. Molyneux A, Kerr R, Stratton I, et al. International Subarachnoid Aneurysm Trial (ISAT) of neurosurgical clipping versus endovascular coiling in 2143 patients with ruptured intracranial aneurysms: a randomised trial. Lancet 2002;360:1267-74

11. Molyneux AJ, Kerr RS, Yu LM, et al. International Subarachnoid Aneurysm Trial (ISAT) of neurosurgical clipping versus endovascular coiling in 2143 patients with ruptured intracranial aneurysms: a randomised comparison of effects on survival, dependency, seizures, rebleeding, subgroups, and aneurysm occlusion. Lancet 2005;366:809-17

12. Ferns SP, Sprengers ME, van Rooij WJ, et al. Coiling of intracranial aneurysms: a systematic review on initial occlusion and reopening and retreatment rates. Stroke 2009;40:e523-29

13. Lawson MF, Newman WC, Chi YY, et al. Stent-associated flow remodeling causes further occlusion of incompletely coiled aneurysms. Neurosurgery 2011;69:598-603

14. Lopes D, Sani S. Histological postmortem study of an internal ca- rotid artery aneurysm treated with the Neuroform stent. Neurosurgery 2005;56:E416

15. Roszelle BN, Babiker MH, Hafner W, et al. In vitro and in silico study of intracranial stent treatments for cerebral aneurysms: effects on perforating vessel flows. J Neurointerv Surg 2013;5:354-60

16. Pierot L, Spelle L, Leclerc X, et al. Endovascular treatment of unruptured intracranial aneurysms: comparison of safety of remodeling technique and standard treatment with coils. Radiology 2009;251: $846-55$

17. Mocco J, Snyder KV, Albuquerque FC, et al. Treatment of intracranial aneurysms with the Enterprise stent: a multicenter registry. J Neurosurg 2009;110:35-39

18. Amenta PS, Dalyai RT, Kung D, et al. Stent-assisted coiling of widenecked aneurysms in the setting of acute subarachnoid hemorrhage: experience in 65 patients. Neurosurgery 2012;70:1415-29

19. Bodily KD, Cloft HJ, Lanzino G, et al. Stent-assisted coiling in acutely ruptured intracranial aneurysms: a qualitative, systematic review of the literature. AJNR Am J Neuroradiol 2011;32:1232-36

20. Pierot L, Cognard C, Spelle L, et al. Safety and efficacy of balloon remodeling technique during endovascular treatment of intracranial aneurysms: critical review of the literature. AJNR Am J Neuroradiol 2012;33:12-15

21. Lodi Y, Latorre J, El-Zammar Z, et al. Single stage versus multistaged stent-assisted endovascular repair of intracranial aneurysms. J Vasc Interv Neurol 2011;4:24-28

22. Saatci I, Yavuz K, Ozer C, et al. Treatment of intracranial aneurysms using the Pipeline flow-diverter embolization device: a single-center experience with long-term follow-up results. AJNR Am J Neuroradiol 2012;33:1436-46

23. Yu SC, Kwok CK, Cheng PW, et al. Intracranial aneurysms: midterm outcome of Pipeline embolization device: a prospective study in 143 patients with 178 aneurysms. Radiology 2012;265:893-901

24. Lubicz B, Mine B, Collignon L, et al. WEB device for endovascular treatment of wide-neck bifurcation aneurysms. AJNR Am J Neuroradiol 2013;34:1209-14

25. Cognard C, Weill A, Spelle L, et al. Long-term angiographic follow-up of 169 intracranial berry aneurysms occluded with detachable coils. Radiology 1999;212:348-56 